\title{
Von der Selbstüberschreitung zur Selbstersetzung
}

\section{Zu einigen anthropologischen Tiefenstrukturen des Transhumanismus}

Zusammenfassung: Der Beitrag will zeigen, dass sich einige ,klassische' philosophische Gedankenfiguren und Selbstdeutungsformen im Transhumanismus wiederfinden, aufheben, verschieben und verschärfen. Insbesondere die Idee einer Konversion des ,alten Adams' zu einem ,neuen' oder ,besseren` Menschen wird (über einige historische Zwischenstationen) zu einem biotechnischen Programm, in dem die gezielte Steuerung der Evolution das Ziel der technischen Optimierung von Menschen wird. Um dies zu zeigen, wird zum einen eine in den Transhumanismus einfließende geistesgeschichtliche Linie skizziert, die man mit der Formel ,vom Konversionsgebot über das Selbsterschaffungspostulat zum Selbstevolvierungsmandat' zuspitzen kann. Zum anderen wird der Transhumanismus vor der Folie des Existentialismus kritisch reflektiert. Zentrale Momente dieses kritischen Vergleichs sind das Pathos des Herstellens im Unterschied zum Pathos des Handelns, das transhumanistischen Selbst-Designs im Unterschied zum existentialistischen Selbst-Entwurf sowie die Rolle der ,Bedingtheiten der conditio humana und der existentiellen Grenzsituationen, denen das transhumane Wesen letztlich entkommen will. Damit soll insgesamt gezeigt werden, dass die transhumanistische Agenda mit der Verbesserung des als defizient eingestuften Wesens Mensch in letzter Konsequenz seine Selbstabschaffung zum Ziel hat.

\section{Einleitung}

Transhumanistische Thesen zum Wesen des Menschen befeuern in den letzten Jahren die Debatte um den Einsatz medizinischer, biotechnologischer und informationstechnischer Mittel zur „Verbesserung“ des Menschen. Im medizinethischen Kontext hat sich der Begriff des ,Enhancement' etabliert, der, als Gegenbegriff zur Therapie verstanden, medizinische Eingriffe zu Optimierungszwecken bezeichnet (siehe zur Übersicht über die Debatte Eßmann/Bittner/Baltes 2011; Gordijn/Chadwick 2008; siehe den Beitrag von Armin Grunwald in diesem Band). Derzeit ist noch nicht allzu viel möglich, einzelne Medikamente scheinen die Aufmerksamkeit 
steigern zu können, auch einige stimmungsaufhellende Präparate sollen über eine Therapie hinaus genutzt werden, um Selbste ,besser ${ }^{\star}$ zu machen (,better than well ${ }^{\text {* }}$ (Elliott 2003). Die Entwicklungen in Informatik und Biotechnologie sowie immer weiter reichende neurotechnologische Eingriffe in das Gehirn ergänzen die recht vage, wenn auch imaginativ offenbar beflügelnde Rede von der technischen ,Optimierung، des Menschen. Ein Grund hierfür mag sein, dass die moralische, kulturelle oder religiöse Perfektionierung in unserer Kulturgeschichte eine so große Rolle spielt - und die Technik eine attraktive Alternative zu bieten scheint: Selbstvervollkommnung ohne eigene Anstrengung.

Der Transhumanismus ist im Kontext der Debatte um die ,Optimierung des Menschen in anthropologischer Hinsicht von besonderem Interesse, weil Transhumanist_innen dafür argumentieren, derartige Entwicklungen aktiv voranzubringen, um die jetzige, als defizient angesehene menschliche Natur zu überwinden. Dabei sollen Kreaturen erzeugt werden, die die aktuelle menschliche Existenzform in verschiedener Hinsicht überschreiten, hin zu Wesen, die von den Akteur_innen der Bewegung ,trans'- oder ,posthuman' genannt werden, weil sie Kerneigenschaften menschlicher Lebensformen (z. B. Endlichkeit, Sterblichkeit, Vulnerabilität) nicht mehr besitzen (siehe Loh 2020, 277-282). Der Transhumanismus stützt sich damit also auf anthropologische Vorannahmen, um sein Programm zu motivieren und zu legitimieren. Bei dem Ausdruck ,transhuman', der hier vorrangig gebraucht werden soll, liegt der Akzent auf der ,Übergangshaftigkeit‘ des zu optimierenden Wesens, wobei der oder die ,Posthumane' zentrale Charakteristika der menschlichen Lebensform bereits hinter sich gelassen hat. Die beiden Begriffe werden trotz dieser Unterscheidung nicht selten synonym verwendet, weil beide die menschliche Natur als einen $\mathrm{zu}$ optimierenden und letztlich zu überwindenden ,Ausgangszustand' für die projektierten bio-, neurooder informationstechnischen Maßnahmen erachten. Von diesen technologisch ausgerichteten Bewegungen ist allerdings der „kritische Posthumanismus“ zu unterscheiden (siehe Loh 2018). Dieser hat nicht die technische „Optimierung“ von Menschen zum Thema, sondern die Kritik an den Vorannahmen des ,klassischen' Humanismus sowie seiner Konzeptionierungen ,des' Menschen und der expliziten wie impliziten moralisch-anthropologischen Agenda, die in diesem Theoriekontext dem (sehr weit gefassten Begriff des) Humanismus zugeschrieben wird und die sich aus der Sicht von kritischen Posthumanist_innen an ,klassischen' (und zu überwindenden) Kategorien und Dichotomien wie Frau/Mann, Natur/Kultur, Subjekt/Objekt usw. ausrichtet (siehe exemplarisch Braidotti 2014 und Braidotti/Hlavajova 2018). Im Folgenden will ich mich ausdrücklich nicht mit dem kritischen Posthumanismus auseinandersetzen, sondern mit dem am Anfang des Paragraphen erwähnten technikgetriebenen Transhumanismus. 
Ausgangspunkt des transhumanistischen Programms ist ein Begriff der menschlichen Natur, der an ihrer Defizienz orientiert ist. Der Oxforder Vordenker des Transhumanismus Nick Bostrom bezeichnet diese als „half-baked beginning that we can learn to remold in desirable ways“ (Bostrom 2003, 4). Diese hemdsärmelig-zupackende Formulierung darf nicht darüber hinwegtäuschen, dass die transhumanistischen Visionen der Überwindung des Menschen an eine Reihe von philosophischen (und theologischen) Gedankenfiguren unterschiedlicher Provenienz anschließen, die den Transhumanismus für seine Verfechter_innen erst attraktiv machen. Der Transhumanismus kann meines Erachtens nicht verstanden werden, wenn wir nicht die Reflexionstraditionen in den Blick bekommen, die der Transhumanismus aufgreift, um sich diese in szientistisch pervertierter Form anzueignen. Ich will dies die anthropologischen ,Tiefenstrukturen' nennen, die die transhumanistischen Vorhaben prägen, auch wenn sie nicht immer explizit gemacht werden. Solange wir diese Tiefenstrukturen nicht erfassen, könnte man den Transhumanismus als eine anthropologisch unterkomplex argumentierende neo-eugenische Bewegung missverstehen. Man darf jedoch nicht übersehen, dass sich in dieser Bewegung verschiedene (alteuropäische) Reflexionstraditionen brechen, von Utopien eines besseren Lebens (Hauskeller 2009; 2012), über Konversionsfiguren (Müller 2014) bis hin zu Erlösungshoffnungen, um nur einige zu nennen. Letztere wurden von Meghan O‘Gieblyn besonders eindringlich beschrieben. O‘Gieblyn erzählt, wie sie nach einer spirituellen Krise ihren Weg vom (evangelikalen) Christentum zum Transhumanismus beschritten hat und dabei im Transhumanismus allerlei religiöse Motive und Versprechungen wiedererkennt, die technisch realisiert werden sollen (O`Gieblyn 2017). Auch Yuval Noah Harari der israelische Historiker, der für seine pointierte Deutung der Genese und Zukunft unserer Zivilisation bekannt geworden ist - spricht von kalifornischen Techno-Religionen, die der eigentliche Antrieb der Technologieentwicklung im Silicon Valley geworden sind: „That's where hi-tech gurus are brewing for us brave new religions that have little to do with God, and everything to to with technology. They promise all the old prizes - happiness, peace, prosperity and even eternal life - but here on earth with the help of technology, rather than after death with the help of celestial beings." (Harari 2015, 351)

Derartige Befunde geben Anlass, einige der philosophischen und theologischen Tiefenstrukturen des Transhumanismus systematisch herauszuarbeiten. Dies kann dazu beitragen, die anthropologischen Vorannahmen des Transhumanismus präziser zu benennen und zu beschreiben, um diese dann kritisch reflektieren zu können. Die Untersuchung dieser Tiefenstrukturen kann damit auch zu einer ethischen Einschätzung beitragen - wenn ,Ethik' nicht nur im engeren Sinne als ,angewandte Ethik' verstanden wird, sondern auch die normative Selbstauslegung in Bezug auf Dynamiken, Programmatiken und Deu- 
tungsmuster umfasst, die in der technischen Zivilisation generiert werden und die individuelle und kollektive Lebensstile prägen sowie bestimmte Handlungsoptionen und die damit zusammenhängenden Selbstdeutungsformen privilegieren oder marginalisieren.

Im Folgenden will ich zweierlei versuchen: Zum einen (2.) will ich zeigen, dass es eine in den Transhumanismus einfließende geistesgeschichtliche Linie gibt, die man mit der Formel ,vom Konversionsgebot über das Selbsterschaffungspostulat zum Selbstevolvierungsmandat‘ zuspitzen kann. Mit dieser geistesgeschichtlichen Linie lässt sich verdeutlichen, dass der Transhumanismus in einer Tradition steht, in der die Idee der Konversion zu einem ,neuen' oder ,besseren Menschen über einige historische Stationen (die nur exemplarisch skizziert werden können) zu einem biotechnischen Programm wird, in dem die gezielte Steuerung der Evolution das Ziel der technischen Optimierung von Menschen wird. Zum anderen (3.) will ich den Transhumanismus im Blick auf den Entwurfscharakter, der das transhumanistische Projekt grundlegend zu charakterisieren scheint - wir können uns selbst auf ein bestimmtes Menschsein hin „frei“ entwerfen -, näher untersuchen, indem ich dessen Agenda vor der Folie des Existentialismus - genaugenommen in Sartres programmatischer Fassung von 1946 - kritisch reflektieren will. Dies bietet sich schon allein deshalb an, weil der Begriff des „Entwurfs“ in Sartres Text mit einem heroisierenden Pathos der Selbsterschaffung verbunden ist, das im Rückgriff auf technomorphe Begriffe und Metaphern ausbuchstabiert wird. Abschließen möchte ich meine Überlegungen mit einem kurzen Resümee (4.).

\section{2}

Die Grundanlage des transhumanistischen Programms sieht, wie schon gesagt, vor, dass eine als defizient angesehene menschliche Natur in einer umfänglichen Weise verbessert werden soll. Welches Ziel genau verfolgt wird, hängt von der jeweiligen Strömung ab: radikale Lebensverlängerung und Unsterblichkeit, sei es durch biotechnologische oder durch informatikgestützte Eingriffe, wie bei dem so genannten ,mind uploading' (siehe hierzu den Beitrag von Tobias Müller in diesem Band), sowie die Verbesserung von Erfahrungsqualitäten (sich besser entfalten können, etwas intensiver erleben können usw.) gehören zum teleologischen Kernbestand des Transhumanismus (siehe Loh 2018). Die Attraktivität dieser technologischen Versprechungen liegt meines Erachtens auch daran, dass der Transhumanismus an ,anthropologische Bedürfnisse‘ anknüpfen kann, die tief in unserer Kulturgeschichte verankert sind und die sich der Transhumanismus szientistisch pervertiert aneignet. Im Folgenden will ich zeigen, dass der Transhu- 
manismus in einer geistesgeschichtlichen Linie steht, die als eine folgenreiche Verschiebung von einem (religiös geprägten), Konversionsgebot‘ über ein (neuzeitlich-anthropologisch verschärftes) ,Selbsterschaffungspostulat' zu einem (technologisch radikalisierten) ,Selbstevolvierungsmandat' beschrieben werden kann. Selbstverständlich kann diese kulturgeschichtliche Linie in diesem Text nur sehr grob und schlagwortartig nachgezeichnet werden.

Als kanonisch in Bezug auf die Idee einer Konversion zu einem besseren Menschen kann eine Stelle im Neuen Testament gelten, in der davon die Rede ist (unter Verwendung einer Metapher aus dem Feld der Bekleidung), dass man den „alten Menschen“ ablegen und den „neuen Menschen“ anziehen soll, um zum neuen Glauben zu finden (Eph 4, 22-24). Dieses Gebot zieht sich durch die Geistesgeschichte, auch in säkularisierten Formen, in denen es eine selbstauferlegte, radikale Neuorientierung bezeichnet. An dieser Stelle soll nur an Sartre erinnert werden, der (in Anlehnung an Trotzkis „permanente Revolution“) von einer „permanenten Konversion“ spricht, die sich das reflektierte Individuum selbst auferlegen solle (Sartre 2005, 28; 822 ff.). Die existentialistische Konversion bedeutet, dass man sich von der „komplizenhaften Reflexion“ frei machen soll, also von einer Art Lebenslüge, die Menschen von sich selbst „entfremdet“ (durchaus auch im Heideggerschen Sinne gedacht, dass man Authentizitätsgewinne erzielt, wenn man sich dem „Man“ entzieht). In einem neuen Entwurf soll der oder die Konvertit_in in authentischer Weise „bei sich selbst und für sich selbst [...] sein“ (ebd., 824f.). Und in Bezug auf den Fluchtpunkt der biotechnischen ,Konversion“ sei an dieser Stelle auch auf den von Peter Sloterdijk „Befehl aus Stein“ genannten Ausgangspunkt aller „Anthropotechnik“ verwiesen, den er aus seiner Lesart des Gedichts „Archäischer Torso Apollos“ von Rainer Maria Rilke entwickelt: „du mußt dein Leben ändern“ (Sloterdijk 2009).

Als geistesgeschichtliches ,missing link‘ zwischen dem Konversionsgebot und seiner bio-anthropotechnischen Aneignung, kann eine Tendenz gelten, die ich mit dem Schlagwort des ,Selbsterschaffungspostulat' bezeichnen und mit der berühmten Passage aus der „Oratio de hominis dignitate“ von Giovanni Pico di Mirandola erläutern will: In diesem Text entwickelt Pico besonders prägnant die Idee, dass Menschen Wesen sind, die ihr ,Wesen' selbst hervorbringen können, weil es konstitutiv für ihr Sein ist, dass sie sich zu dem machen können, was sie sein wollen (Pico della Mirandola 1988). ${ }^{1}$ Bei Pico ist es sogar göttliches Gebot, dass der Mensch sich in seiner Freiheit selbst zum ,Menschen' macht. Als Geschöpf ist ,der‘ Mensch zunächst unspezifisch zwischen Gott und den Tieren

1 Auch Ralf Becker sieht Picos Text als zentral für das Motiv der Selbstüberschreitung ,des“ Menschen an: Ralf Becker (2015). 
platziert. Doch analog zur großen Schöpfung Gottes, die bei Pico in auffällig technisch-handwerklicher Metaphorik beschrieben ist (siehe Meyer-Drawe 1996, 52ff.), wird der Mensch der Schöpfer im Kleinen, nämlich zum Schöpfer seiner selbst. Damit kann sich ,der' Mensch sich nach dem von ihm selbst konzipierten Wesen selbst ,herstellen' (um es mit einer Häufung von Reflexivpronomina auszudrücken). Picos Rede über die Würde des Menschen ist daher ein anthropologischer Schlüsseltext, weil wir hier ein Selbstverständnis erkennen können, das von einem neuen humanen Selbstbewusstsein zeugt. Dass sich das Motiv, dass der Mensch das „nicht-festgestellte Thier“ ist (wie es Nietzsche bekanntermaßen ausdrücken wird), das aufgrund dieser Konstitution die Aufgabe hat, sich selbst erst ,machen' zu müssen, schon in diesem Renaissance-Text mit einem technischen Vokabular verbindet, ist kein Zufall. Das technische Herstellen ist der konkrete Anschauungsraum, in dem die Selbsterschaffung nachvollzogen werden kann.

Und vor diesem Hintergrund ist es dann geradezu folgerichtig, die Selbsterschaffung nicht nur moralisch oder kulturell zu verstehen, sondern als biotechnologisches Projekt zu interpretieren. Auch wenn die Schaffung künstlicher androider Wesen schon seit der Antike zum Imaginationsraum erträumter technischer Machbarkeiten gehört (Müller/Liggieri 2019, 3-14), ändert sich der Selbstdeutungshorizont mit der beginnenden Industrialisierung und der Ausdifferenzierung der Lebenswissenschaften und der Medizin. Es ist bezeichnend für die Reflexion über die neue Rolle der Biotechnologien, dass am Anfang des 19. Jahrhunderts sowohl der Briefroman „Frankenstein, or The Modern Prometheus“ von Mary Wollstonecraft Shelley und der zweite Teil des „Faust“ von Johann Wolfgang von Goethe entstehen. Beide Texte erzählen von der biotechnischen Schöpfung menschenähnlicher Kreaturen mittels biotechnologischer Experimente; wobei im „Frankenstein“ ein ,untermenschliches“ (das ,Monster') und im „Faust“ ein ,übermenschliches‘ Wesen (der Homunculus) geschaffen wird. Dass die Biotechnologie in diesen literarischen Szenarien die ,menschliche Norm` unter- und überschreitet, kann man als die inhärente ,Logik ${ }^{\star}$ der modernen Wissenschaft und Technik verstehen, die in der baconianischen Tradition des ,plus ultra' steht, das bislang Menschenmögliche zu transzendieren. In den Texten von Shelley und Goethe wird die Technik noch nicht verwendet, um den Schöpfer selbst zu verbessern. Aber es wird die Bühne dafür bereitet, die Technologie nicht nur zur Erschaffung eines anderen Wesens zu nutzen, sondern auch aus sich selbst ein neues Wesen zu machen, das die bisherige conditio humana transzendiert.

Im „Faust“ wird die Transformation von der ,Fremdschöpfung“ zur ,Selbstschöpfung‘ explizit zum Thema gemacht. Der Ausgangspunkt für die Projektierung der Kreation des Homunculus ist die Überwindung der als ,schmutzig` an- 
gesehenen sexuellen Zeugung, aus der Menschen herkömmlicherweise entstehen: In der Vision der Figur Wagner, des Biotechnologen avant la lettre, soll der Mensch „künftig höhern, höhern Ursprung haben“ (Goethe 1981, V. 6847). Und genau diese Veredelung des Menschen durch die ,reine“ Laborschöpfung führt zum Bedürfnis, dass der Herstellende auch sich selbst herstellen will: „Und so ein Hirn, das trefflich denken soll / Wird künftig auch ein Denker machen.“ (Ebd., V. 6869f.) Der bestmögliche Biotechnologe hat sich selbst produziert, bevor er andere Kreaturen schafft. Sein künstlich hergestelltes (oder aufgerüstetes) Gehirn ist die Voraussetzung für seine Schöpfungstätigkeit. Die Laboratoriums-Szene ist damit nicht nur ein wissenschaftstheoretischer, sondern auch ein anthropotechnischer Echoraum (siehe Liggieri 2014). Der Mensch als Homo faber richtet sich nicht nur die Welt technisch ein, zur ,Metaphysik‘ des Homo faber gehört auch das Sich-sich-selbst-Verdanken-Wollen (Müller 2010), eine Grundfigur moderner Technik, die Günther Anders besonders präzise beschrieben hat: Der Homo faber will nicht geworden oder geboren sein, „sondern wünscht, sich als sein eigenes Produkt sich selbst zu verdanken“ (Anders 1956, 325; siehe auch den Beitrag von Christina Schües in diesem Band).

Dieses seit Pico technisch transformierte Selbsterschaffungspostulat ist nun die Voraussetzung für das, was ich ,Selbstevolvierungsmandat' nennen will und mit dem wir die unmittelbare Vorgeschichte des Transhumanismus erfassen können. Seit Darwins Evolutionstheorie wurde der Mensch in verschiedenen Kontexten als „Zwischenwesen“ („creatures of twilight“) verstanden, dessen Leben (in Anlehnung an Hobbes) als „nasty brutish and short“ bezeichnet wurde (siehe Coenen 2009; Coenen et al. 2010). So unterschiedliche Proponenten wie Julian Huxley oder H. G. Wells waren wie viele Eugeniker_innen von der Evolutionstheorie befeuert: ,Der` Mensch galt als zukunftsoffen, gestaltbar, planbar wenn es gelingen könnte, die evolutionsbiologischen Prozesse entsprechend zu lenken. Die Arbeit am Menschen, die bislang die Natur vorgenommen hatte, durfte der Mensch nun mit seiner Technik fortzusetzen hoffen. Darwin selbst hatte am Ende des „Descent of Man“ derartige Erwartungen formuliert: „Es ist begreiflich, daß der Mensch einen gewissen Stolz empfindet darüber, daß er sich, wenn auch nicht durch seine eigenen Anstrengungen, auf dem Gipfel der organischen Stufenleiter erhoben hat; und die Tatsache, daß er sich so erhoben hat, anstatt von Anfang an dorthin gestellt zu sein, mag ihm die Hoffnung auf eine noch höhere Stellung in einer fernen Zukunft erwecken.“ (Darwin 2005, 274) Nach diesen Sätzen unterstreicht Darwin allerdings, dass der Mensch trotz aller hohen (gottähnlichen) geistigen Fähigkeiten doch auch unverkennbar dem Animalischen verhaftet bleibe. Dies ist bekanntlich als ,Darwinsche Kränkung‘ in die Kulturgeschichte eingegangen. Doch es war eben auch derselbe Darwin, der die Hoffnung auf eine noch höhere Stellung im Kosmos artikuliert (wiewohl er einen 
biotechnologischen Beitrag hierzu vermutlich noch nicht im Blick hatte). Dies ändert sich mit den Darwinisten und Eugenikern der ersten Stunde, die sich von den neuen Aussichten auf eine höhere Existenz berauschen ließen - und als biotechnischen Auftrag zu interpretieren begannen. Bis heute scheint sich aus Darwins Befund ein transhumanistisches Argument geradezu zwingend zu ergeben: Der Mensch ist dank seines Wissens und Könnens dazu verpflichtet, seine Evolution selbst in die Hand zu nehmen. Um es mit einer Kant-Parodie auszudrücken: Wir befinden uns von nun an in einer Situation selbstverschuldeter Unverbessertheit - und sollen Mut haben, uns unserer Technologien zu bedienen.

Nicht von ungefähr fällt die erste eugenische Begeisterung in die Zeit, in der Nietzsche mit seinem „Übermenschen“ einen Begriff prägt, der ebenfalls die Überwindung der aktuellen menschlichen Lebensform proklamiert: „Der Mensch ist Etwas, das überwunden werden soll“ (Nietzsche 2014, 14). Nietzsches Übermensch lässt sich natürlich nicht auf die biotechnologische Selbststeigerung reduzieren. Doch gibt er mit seiner Rede von der ,Brückenexistenz“ des Menschen zwischen Tier und Übermensch (ebd., 16) das anthropologische Stichwort, das den Menschen als einen bloßen Übergang zu seiner (wie auch immer gearteten) ,höheren` oder ,besseren' Existenzform versteht. Explizit sagt Nietzsche provokant, dass der Mensch kein „Zweck“ ist (u.a. gegen Kant gerichtet), sondern eine „Brücke“ (ebd., 14). Nietzsche eröffnet in diesem Zusammenhang selbst einen Diskursraum, der darwinistisch-transhumanistisch interpretiert werden kann: „Was ist der Affe für den Menschen? Ein Gelächter oder eine schmerzliche Scham. Und ebendas soll der Mensch für den Übermenschen sein: ein Gelächter oder eine schmerzliche Scham.“ (Ebd.)

Daher gibt es auch eine Debatte unter Transhumanist_innen über die Frage, ob und inwiefern der Transhumanismus in der Tradition von Nietzsches Übermenschentum anzusiedeln ist (Bostrom 2006; Sorgner 2009; Tuncel 2017). Nietzsches Scham und Gelächter über den jetzigen Menschen kehren nun über die Fallhöhe der posthumanen Existenz wieder. Es ist typisch für transhumanistische Positionen, dass sie neue Formen des Erlebens imaginieren, die weit über das für heutige Menschen Übliche und Mögliche hinausreichen. Um an dieser Stelle nur ein Beispiel zu nennen: „Your experiences seem more vivid“, schreibt Nick Bostrom in einer kleinen transhumanistischen Utopie, , [w] hen you listen to music you perceive layers of structure and a kind of musical logic to which you were previously oblivious; this gives you great joy [...]. Instead of spending four hours each day watching television, you may now prefer to play the saxophone in a jazz band and to have fun working on your first novel [...]. You still listen to music music that is to Mozart what Mozart is to bad Muzak.“ (Bostrom 2006, 111f.) Wir werden also Musik hören können, die so weit von Mozart entfernt ist wie Mozart von schlechter Fahrstuhlmusik in unseren Tagen. Mozart wird bei den transhu- 
manistischen Zukunftsmusikern genau das auslösen, was Nietzsche mit dem künftigen Blick des Übermenschen auf seine lächerliche menschliche Vorform vermutet: Gelächter und schmerzliche Scham.

Diese verächtliche Perspektive auf die menschliche Natur ist der Motor für die Ansätze, die Evolution zu korrigieren, um die Höherentwicklung des Menschen zu garantieren und gezielt zu steuern (siehe hierzu auch den Beitrag von Armin Grunwald in diesem Band). Dies drückt sich in den markigen Aussagen von Biotechnologen aus, die seit ein paar Jahren durch die Gazetten geistern. So sagt Tim Knight etwa: „Der genetische Code ist 3,6 Milliarden Jahre alt. Es wird Zeit, ihn neu zu schreiben. “ - Während sich Freeman Dyson mit folgendem Satz zitieren lässt: „This notion implies not only that humanity is now fully in command of its own destiny, it implies also that we are no longer subject to the haphazard, cumbersome, and often inefficient ways of evolution." Und Stefan Lorenz Sorgner, der im deutschsprachigen Raum sicher der bekannteste bekennende Transhumanist ist, schreibt in seinem Plädoyer für einen ,Nietzscheanischen Transhumanismus': „Es ist naiv, davon auszugehen, dass der Homo sapiens sapiens in 6 Millionen Jahren noch immer existieren wird. Spezies müssen sich auf beständige Weise neu anpassen. Entweder eine Spezies passt sich an oder sie stirbt aus. Deshalb ist es notwendig, beständig auf neue Techniken zurückzugreifen, und diese zu entwickeln. Dies ist die entscheidende Grundannahme, die von allen Transhumanisten geteilt wird.“ (Sorgner 2019) Damit ist nun der Boden für das transhumanistische Programm bereitet, das aus der geistesgeschichtlichen Linie vom Konversionsgebot über das Selbsterschaffungspostulat beim Selbstevolvierungsmandat angekommen ist. Vor diesem Hintergrund wird die eingangs formulierte These (hoffentlich) verständlich: Der Transhumanismus ist eine szientistisch-technische Pervertierung eines ,alten' menschlichen Selbsttranszendierungsbedürfnisses.

An dieser Stelle will ich diese Reflexionstradition verlassen und mich einem Aspekt zuwenden, der in der Forschung über den Transhumanismus bislang noch nicht behandelt wurde und (soweit ich sehe) auch in der Selbstreflexion der Vertreter_innen der transhumanistischen Bewegung nicht thematisiert wird: der an den Existentialismus erinnernde ,Entwurfscharakter ${ }^{\star}$ der menschlichen Existenz. Im folgenden zweiten Teil meines Beitrages will ich nachzeichnen und diskutieren, ob man dem Transhumanismus eine ,kryptoexistentialistische“ Tendenz zuschreiben kann und ob und inwiefern sich diese von Programm und Anspruch des ,klassischen“ Existentialismus unterscheidet. Das Ziel ist auch in diesem Teil, eine philosophisch-anthropologische Tiefenstruktur freizulegen, um den Transhumanismus mit Hilfe einer anderen Perspektivierung kritisch reflektieren zu können. 
Auf den ersten Blick könnte man den Transhumanismus als dem Existentialismus völlig entgegenlaufend verstehen. Denn wenn durch die biotechnische Optimierung Angst, Verzweiflung und Schuld ihre existentielle Kraft einbüßen sollen, und wenn auch die Herausforderung des eigenen Todes nicht mehr zu jenem orientierungssuchenden Ringen führen muss, das den Existentialismus charakterisiert - gehört doch die medizintechnologische ,Abschaffung' des Todes, dieser vermutlich größten Provokation des Menschseins zu den Kernzielen des Transhumanismus -, dann dürften die Probleme des menschlichen Daseins gelöst sein. Dürfen wir uns also den Transhumanen als einen glücklichen Menschen vorstellen?

Schauen wir uns das näher an: Auch der Existentialismus besteht aus einer Bandbreite von Positionen und speist sich überdies aus einer spezifischen Theoriegeschichte, die eng mit der politischen Situation um 1945 und dem Zusammenbruch der Vertrautheitsstruktur überkommener religiöser und philosophischer Ordnungsrahmen verknüpft ist. Ich will mich in diesem Kontext lediglich auf den Existentialismus in seiner programmatischen Ausprägung beziehen, die ihm Sartre in seinem bekannten Text „Ist der Existentialismus ein Humanismus?“ von 1946 gegeben hat (Sartre 1975). Allein schon die Ähnlichkeiten im programmatischen und popularisierenden Stil prädestinieren diesen Text als Folie für die transhumanistischen Verlautbarungen.

Eine der bekannten Thesen, für die Sartre in seinem Essay wirbt, ist jene, dass die Existenz der Essenz vorausgehe. Sartre erklärt sein Philosophem bemerkenswerterweise unter Verwendung technischer Beispiele: „Betrachten wir ein Artefakt, zum Beispiel ein Buch oder ein Papiermesser, so ist dieser Gegenstand von einem Handwerker angefertigt worden, der sich von einem Begriff hat anregen lassen; er hat sich auf den Begriff Papiermesser bezogen und zugleich auf eine vorher bestehende Technik der Erzeugung, welche zu dem Begriff gehört und im Grunde ein Rezept ist." (Ebd., 9f.) Diesen Übergang vom Begriff zum Artefakt, von der Definition zum technischen Produkt erklärt Sartre mit Hinweisen auf den metaphysischen Hintergrund der Voraussetzungen von Existenz überhaupt: „Wir haben also hier ein technisches Bild der Welt, in der, kann man sagen, die Erzeugung der Existenz vorausgeht. Wenn wir einen Schöpfer-Gott annehmen, so wird dieser Gott meistens einem höherstehenden Handwerker angeglichen [...]. Demnach ist der Begriff Mensch im Geiste Gottes dem Begriff Papiermesser im Geiste des Handwerkers anzugleichen, und Gott erzeugt den Menschen nach Techniken und einem Begriff, genau wie der Handwerker ein Papiermesser nach einer Definition und einer Technik anfertigt.“ (Ebd., 10) 
Dieses theologische Erbe will Sartre naturgemäß hinter sich lassen, die technische Hintergrundmetaphorik bleibt aber prägend für den Existentialismus in dieser Form: Menschen als Homines fabri sind gleichzeitig die sich selbst herstellenden Wesen. Hans Blumenberg hat diesen Zug des Existentialismus daher „autotechnisch“ genannt (Blumenberg 1953). Was damit gemeint ist, kann man an folgender Passage aus Sartres Text verdeutlichen: „Was bedeutet es hier, daß die Existenz der Essenz vorausgeht? Es bedeutet, daß der Mensch zuerst existiert, sich begegnet, in der Welt auftaucht und sich danach definiert [...]. Also gibt es keine menschliche Natur, da es keinen Gott gibt, um sie zu entwerfen. Der Mensch ist lediglich so, wie er sich konzipiert - ja nicht allein so, sondern wie er sich will und wie er sich nach der Existenz konzipiert [...]. Der Mensch ist nichts anderes als wozu er sich macht.“ (Sartre 1975, 11)

Es lässt sich leicht erkennen, dass der Transhumanismus diesem Ansatz nicht fernsteht. Der oder die Transhumane lässt sich ebenfalls als ein Wesen beschreiben, das sich selbst gemacht hat bzw. sich selbst gemacht zu haben wünscht. Der oder die Transhumane schafft sich selbst nach seinem oder ihrem eigenen Bild und nach Maßgabe des technisch Möglichen (in einer imaginierten mehr oder weniger fernen Zukunft). Insofern findet sich die Selbstmodellierung nach dem Vorbild der Herstellung eines technischen Produkts auch im Transhumanismus wieder - allerdings in einer deutlichen Verschärfung. Im Fall der transhumanen Lebensform geht die Existenz der Essenz folgendermaßen voraus: Die als unzureichend und biologisch hinfällig verstandene Existenz bedingt eine Essenz, die durch das biotechnologisch Mögliche festgelegt ist. Dies kommt z. B. in der Idee einer „evolutionary heuristic“ zum Ausdruck (Bostrom/Sandberg 2008). Das bedeutet: Das, was die Evolution auf die Dauer ohnehin wohl ,weiterentwickeln' würde - weil die menschliche Natur eben ein ,half-baked beginning‘ darstellt -, soll mit biotechnologischen Mitteln forciert und beschleunigt werden.

Im transhumanistischen Entwurf ist die künftige, ,höhere‘ Existenzform als Blaupause für die Optimierung der jetzigen Existenz verstanden. Insofern wäre der Transhumanist im Vergleich zum Existentialisten in seiner Wahlmöglichkeit deutlich festgelegt: Er kann letztlich nur denjenigen Entwurf seiner selbst wählen, der biotechnologisch produziert werden kann. Die Selbstwahl wird im Lichte von Technoimaginationen gefällt. Dabei spielt es keine Rolle, ob eine Selbstverbesserung technisch möglich ist oder sein wird, sondern es kommt darauf an, das zu Verbessernde im Horizont bio- und informationstechnisch möglicher Eingriffe zu sehen. Dabei ist zunächst bemerkenswert, dass die Logik der technischen Verbesserung und Verfeinerung von Artefakten auch auf die subjektiven Erlebnisqualitäten übertragen wird. Bostroms Idee, bessere Musik zu hören oder zu machen, speist sich letztlich aus dem Vorbild der Innovationen in der Musikgeräteindustrie. 
Die Verbesserungen, die wir in der Produktion von Verstärkern und Lautsprechern beobachten können, sind das Muster für die Verbesserung menschlicher auditiver Fähigkeiten. Einer der Gründe, warum sich Transhumanisten die Verbesserung von Erlebnisqualitäten zu ihren Zielen gemacht haben, könnte die Übertragung der exponentiell steigenden Entwicklungskurven der digitalen Welt auf die menschliche Disposition sein. Vielleicht ist es kein Zufall, dass Ray Kurzweil in seiner prätranshumanistischen Phase mit seinen Synthesizern (Kurzweil Music Systems) Artefakte produziert hat, die auf die Erweiterung musikalischer Klangwelten zielen. Hier könnte die Idee der transhumanistischen Erweiterung der musikalischen Erlebnisfähigkeit als Komplement zur Verbesserung der musikindustriellen Artefakte einen ,Resonanzraum' gefunden haben.

Auch Sartres nächster Punkt ist in Bezug auf die Deutung des Transhumanismus aufschlussreich: Er unterstreicht, dass der Mensch, der sich zu dem machen kann, zu dem er sich machen will, ein Wesen ist, das zu wählen in der Lage ist. Menschen wählen nach Sartre den Optimalentwurf von sich selbst als Mensch oder wie Sartre es ausdrückt: „So bin ich für mich selbst und für alle verantwortlich, und schaffe ein bestimmtes Bild des Menschen, den ich wähle; indem ich mich wähle, wähle ich den Menschen.“ (Sartre 1975, 13) Dieses Moment des Existentialismus kann dazu beitragen, eine Antwort auf die Frage zu finden, warum der Transhumanismus überhaupt als solcher auftritt (also als Transhumanismus). Es wäre ja denkbar, dass die transhumanistischen Visionäre einfach individuelle Gründe anführen und sagen könnten: Ich, Nick, oder ich, Ray, will z. B. etwas Komplexeres als Mozart hören oder länger leben. Doch die Transhumanist_innen beziehen sich gerade nicht auf individuelle Präferenzen, sie verfolgen vielmehr eine Argumentationslinie, die eine unfertige menschliche Natur gegen ein optimiertes Wesen ausspielt, das das Menschsein irgendwann hinter sich gelassen haben wird. Die individuelle Verbesserung soll mit der Höherwertigkeit der neuen Existenzform legitimiert werden. Im Vergleich mit der existentialistischen Wahl könnte man den transhumanistischen Ansatz folgendermaßen begreiflich machen: Indem z.B. Bostrom sich eine künftige bessere Erlebnisfähigkeit wünscht, wählt er nicht eine individuelle Präferenz, sondern ein Wesen, das es noch nicht gibt, das er sich aber herbeisehnt. In der Wahl der Optimierung seiner individuellen Disposition wählt Bostrom die Überschreitung des Menschen als solchen, wählt sich selbst als Transhumanen. Wenn Sartre in dem ihm eigenen Pathos (und im Rückgriff auf Francis Ponge) unterstreicht, dass der „Mensch die Zukunft des Menschen“(ebd., 17) sei, dann würde ein/e Transhumanist_in ihm in diesem Punkt widersprechen und sagen: Der Mensch muss nicht unbedingt die Zukunft des Menschen sein. Wir können auch die Wahl treffen, kein Mensch mehr sein zu wollen, sondern ein Wesen, das Mensch war. 
Sartres Prämisse, dass es keine festumrissene menschliche Natur gibt (ebd., 21), kann der Transhumanismus ebenfalls nicht teilen: Denn der Transhumanismus muss gerade eine einigermaßen klar umrissene menschliche Natur behaupten, denn sonst gäbe es nichts, was überwunden werden soll. Zudem muss sich die technische ,Optimierung، auf bestimmte Vorstellungen der menschlichen Natur stützen, um ebendiesen Stand der Natur ,optimieren' zu können. Ein/e Existentialist_in, auf der anderen Seite, hätte ihrer- oder seinerseits Skepsis gegenüber dem transhumanistischen Programm, weil Existentialist_innen nach Sartre grundsätzlich „nicht an den Fortschritt glauben“, denn: „der Fortschritt ist eine Verbesserung; der Mensch ist immer derselbe einer Situation gegenüber, die sich verändert, und die Wahl bleibt immer eine Wahl inmitten einer Situation.“ (Ebd., 30)

Auch wenn wiederum der oder die Transhumanist_in nicht leugnen können wird, dass sich die (politische, historische, soziale usw.) Situation von Menschen in einer Weise ändern kann, dass eine Änderung der Selbstwahl verlangt sein könnte, würde man aus einer transhumanistischen Perspektive vermutlich sagen, dass die biotechnologische Umformung gerade $\mathrm{zu}$ einer gewissen Autarkie gegenüber dieser Art von Situationen führen soll. Während der oder die Existentialist_in seinem oder ihrem Entwurf üblicherweise angesichts der Herausforderungen der conditio humana (wie z.B. der eigene Tod, die Krankheit, die Endlichkeit) wählt, wollen Transhumanist_innen sich selbst in Bezug auf künftige Wesen wählen, für die idealerweise die ,klassischen' Grenzsituationen überwunden sind und für den Selbstentwurf keine Rolle mehr spielen. Während die Freiheit des Sich-selbst-Entwerfens im Existentialismus an Bedingtheiten der menschlichen Existenz geknüpft sind und sich gerade in dem Bejahen der Endlichkeit die Freiheit, eine Selbst-Wahl zu treffen, manifestiert, verkennen Transhumanist_innen diese Voraussetzung menschlicher Freiheit und propagieren die Vorzüge einer alternativen Lebensform, die sich nicht mehr in Bezug auf die Bedingtheiten zu entwerfen sollen braucht. So sagt beispielsweise Ray Kurzweil in einem Interview: „Der Tod ist schwer vorstellbar, denn unsere Selbstwahrnehmung, unser Bewusstsein kommt uns nicht vergänglich, sondern dauerhaft vor. Trotzdem müssen wir beobachten, dass Menschen nicht ewig leben [...]. Es wird allenthalben bestritten, dass der Tod furchterregend und tragisch ist - von dem Leid, das der Prozess des Sterbens bringt, ganz zu schweigen. Stattdessen wird das Problem rationalisiert, indem man sagt, der Tod sei gut. Und man hängt sehr an dieser Rationalisierung, weil sie es uns erlaubt, weiterzumachen im Angesicht der heraufziehenden Tragödie. Solange wir keine Alternative hatten, war das vernünftig. Heute aber haben wir eine Alternative.“ (Hülswitt/Brinzanik 2010, 17) Die Alternative, von der Kurzweil hier redet, ist die „Singularität“, die Menschen über mehrere Innovationsstufen auf biochemischer, neurotechnologischer und 
informationstechnologischer Ebene unsterblich machen soll. In Bezug auf die im ersten Kapitel entwickelte geistesgeschichtliche Linie ist interessant, dass Kurzweil hier - man erinnere sich an Nietzsche - mehrere „Brücken“ unterscheidet, (ebd., 18; siehe auch Kurzweil 1999) in denen die von ihm angestrebte höhere posthumane Existenzform erreicht werden soll.

An dieser Stelle könnte man pointiert fragen, ob nur Menschen Existentialist_innen sein können, Transhumane aber nicht. Denn es geht ja gerade darum, ein Wesen zu kreieren, das gar nicht mehr in der misslichen Lage ist, sich in Bezug auf die Herausforderungen der conditio humana entwerfen zu müssen, da diese in der transhumanen Lebensform ja überwunden werden sollen. Doch kann man die Bedingungen der Existenz auch als Transhumane/r wirklich hinter sich lassen? Hannah Arendt hat ihr Buch „Vita activa“ mit einer Reflexion über die „conditio humana“ eingeleitet. Dabei arbeitet sie heraus, dass die Rede von ,der menschlichen Natur zwar problematisch, aber dass es doch stimmig sei, über die $B e$ dingtheiten der menschlichen Existenz zu reden, wie z.B. Natalität, Mortalität, Pluralität und Weltlichkeit (Arendt 2002, 16 ff.). Im Zuge dessen macht sie ein Gedankenspiel: Sie imaginiert eine in radikaler Weise technisch transformierte (post)humane Existenzform, die auf einem anderen Planeten lebt: „Dies würde heißen, daß die Menschen ihre Leben den irdisch-gegebenen Bedingungen ganz und gar entziehen und es gänzlich unter Bedingungen stellen, die sie selbst geschaffen haben.“ (Ebd., 20) Die Formulierung, ,das Leben unter selbst geschaffene Bedingungen zu stellen', erinnert ohne Zweifel an das Ziel der transhumanistischen Agenda. Allerdings sagt Arendt nun: „Der Erfahrungshorizont eines solchen Lebens wäre vermutlich so radikal geändert, daß das, was wir unter Arbeiten, Herstellen, Handeln, Denken verstehen, in ihm kaum noch einen Sinn ergäbe. Und doch kann man kaum leugnen, daß selbst diese hypothetischen Auswanderer noch Menschen blieben; aber die einzige Aussage, die wir über ihre Menschennatur machen könnte, wäre, daß sie immer noch bedingte Wesen sind, wiewohl unter solchen Verhältnissen die menschliche Bedingtheit nahezu ausschließlich das Produkt von Menschen selbst wäre.“ (Ebd.)

Das klingt nach Protoposthumanismus in Reinform: Das neue Wesen wäre ein Wesen, das in ganz anderer Weise denkt und handelt als jetzige Menschen. Eine solche Transgression würde die Redeweise ,posthuman` zweifelsohne rechtfertigen (auch wenn sich Arendt natürlich in keiner Weise einem solchen Programm anschließen würde, allein schon wegen der Bedeutung der Natalität in ihrer Anthropologie). Gleichzeitig können wir mit Arendt aber auch einen blinden Fleck des Transhumanismus identifizieren: Auch wenn dieser in der Lage sein sollte, die menschliche Natur entsprechend umfassend umzuformen, würde doch ein Wesen entstehen, das weiterhin bedingt bleibt und in dieser Bedingtheit seine Existenzform ausbildet. Das heißt: Es könnte also sein, dass die conditio posthumana 
sich auf einer prinzipiellen Ebene von der conditio humana gar nicht unterscheidet - weil der oder die Posthumane letztlich ein bedingtes Wesen bleibt, auch wenn es die selbstgeschaffenen Bedingungen sind. Oder anders formuliert: Auch wenn einige Momente der conditio humana überwunden wären, würde das Faktum der Bedingtheit in anderer Form auch die conditio posthumana prägen so dass mit der Überwindung des Menschlichen möglicherweise gar nichts gewonnen wäre. Nach Arendt könnte sich die Situation sogar insofern verschärfen, als der oder die Transhumanist_in künftig ihren oder seinen selbst geschaffenen Bedingungen unterliegen wird. Wie auch immer diese Bedingtheiten aussehen werden, der oder die Transhumane wäre zwar nur noch von ihrem oder seinem eigenen technischen Entwurf abhängig, würde aber das Faktum der Bedingtheit per se nicht beseitigen (siehe auch den Beitrag von Christina Schües in diesem Band).

Nach diesem Exkurs zu Arendt und den Bedingungen der menschlichen Existenz wieder zurück zu Sartre. Einen weiteren zentralen Unterschied von Existentialismus und Transhumanismus kann man folgenermaßen umreißen: Im Existentialismus gibt es ein Pathos des Handelns, während es im Transhumanismus ein Pathos des Herstellens gibt. ,Pathos des Handelns` besagt, dass Menschen sich in ihrem Handeln konstituieren, meist in einem weiten Sinne als politisches Handeln oder Handeln in Grenzsituationen verstanden: Sie sind im emphatischen Sinne das, was sich in ihrem Handeln zeigt, weil sich im Handeln das ,wahre Selbst‘ zeigt. Das ,Pathos des Herstellens‘ wiederum bedeutet, dass ,der' Mensch sich in dem, was er technisch aus sich macht, konstituiert. Die Selbsttransformation erfolgt dabei in einem technoimaginativen Selbstdeutungsraum. Man könnte also sagen: Hier steht dem existentialistischen Selbst-Entwurf das transhumanistische Selbst-Design gegenüber. Oder anders ausgedrückt: Während der Existentialismus (idealistische) Selbstsetzungsfiguren im Entwurfs-Begriff zu aktualisieren und zu radikalisieren sucht, könnte man das Programm Transhumanismus in der Sprache des Deutschen Idealismus folgendermaßen beschreiben: Statt um Selbstsetzung geht es letztlich um Selbstersetzung. Während der existentialistische Selbst-Entwurf auf eine Realisierung eines emphatisch verstandenen Menschseins angesichts von Kontingenzen, Bedingtheiten und Grenzsituationen ausgerichtet ist, hat das transhumanistische Selbst-Design das Ziel, ein Wesen zu kreieren, das nicht mehr Mensch sein muss.

Trotz all dieser Unterschiede finden sich in dem Moment der ,Überschreitung“ wieder auffällige Ähnlichkeiten in den Agenden von Existentialismus und Transhumanismus. Am Ende seines Textes konturiert Sartre seinen Humanismus nämlich wie folgt: „Aber es gibt einen andern Begriff des ,Humanismus“, welcher im Grunde genommen dies bedeutet: Der Mensch ist dauernd außerhalb seiner selbst; indem er sich entwirft und indem er sich außerhalb seiner verliert, macht 
er, daß der Mensch existiert, und auf der andern Seite, indem er transzendente Ziele verfolgt, kann er existieren; der Mensch ist diese Überschreitung, und so befindet er sich im Herzen, im Mittelpunkt dieser Überschreitung. “ (Sartre 1975, 35) Und er unterstreicht, dass er den Begriff ,transzendent" nicht theologisch verstehen will, sondern eben im Sinne der „Überschreitung“ durch die Fähigkeit des permanenten Selbst-Entwurfs bzw. der permanenten Konversion, um den Begriff aus den „Entwürfen für eine Moralphilosophie“ noch einmal aufzugreifen. Nur ein kleiner Schritt wäre es gewesen, diesen Begriff des Transzendierens in einer Vorsilbe zu konservieren - und von einem Trans-Humanismus zu sprechen.

\section{4}

Mit meinem Beitrag wollte ich zum einen herausarbeiten, wie sich bestimmte ,klassische، philosophische Gedankenfiguren und Selbstdeutungsformen im Transhumanismus wiederfinden, aufheben, verschieben oder verschärfen, um auf diese Weise einige anthropologische Tiefenstrukturen des Transhumanismus freizulegen. In der sehr knappen Skizze einer geistesgeschichtlichen Linie, die ich mit der Formel ,vom Konversionsgebot über das Selbsterschaffungspostulat zum Selbstevolvierungsmandat‘ zugespitzt habe, wollte ich zeigen, wie der Transhumanismus auf ,alte، anthropologische Bedürfnisse eine szientifisch-technische Antwort bieten will. Die Freilegung dieser geistesgeschichtlichen Linie kann zur kritischen Evaluierung des transhumanistischen Programmes beitragen, indem man das im Menschsein verankerte Bedürfnis der Selbsttranszendenz im Blick auf die vom Transhumanismus propagierten technische Verengung dieses Selbstüberschreitungsbedürfnisses diskutieren kann - eine Verengung des Begriffs des Mensch-seins, die der Titel eines Artikels in der New York Times, der sich mit den transhumanistischen Tendenzen im Silicon Valley auseinandersetzt, sehr schön auf den Punkt bringt: „Merely Human? That’s So Yesterday“ (Vance 2010).

Auch die Relektüre der existentialistischen Programmschrift „Ist der Existentialismus ein Humanismus?“ im Lichte der transhumanistischen Agenda kann, wie gezeigt, dazu beitragen, charakteristische Merkmale des Transhumanismus herauszuarbeiten und kritisch zu reflektieren. Zentrale Momente waren das Pathos des Herstellens (im Unterschied zum Pathos des Handelns), des transhumanistischen Selbst-Designs (im Unterschied zum existentialistischen SelbstEntwurf) sowie die Rolle der ,Bedingtheiten“ der conditio humana und der existentiellen Grenzsituationen, denen das transhumane Wesen letztlich entkommen will. Deutlich geworden sein dürfte auch das merkwürdige Verständnis ,des Menschen in der transhumanistischen Agenda, die mit der Verbesserung des als 
defizient eingestuften Wesens in letzter Konsequenz die Selbstabschaffung zum Ziel hat.

\section{Literatur}

Anders, Günther (1956): Die Antiquiertheit des Menschen. Bd. 1: Über die Seele im Zeitalter der zweiten industriellen Revolution, München.

Becker, Ralf (2015): Der Mensch will über den Menschen hinaus. Hinweise zu einer Ideengeschichte des homo creator, in: Hartung, Gerald/Herrgen, Matthias (Hg.): Interdisziplinäre Anthropologie 3, Berlin, 165-186.

Blumenberg, Hans (1953): Technik und Wahrheit, in: Actes du XI. Congrès International de Philosophie, Bruxelles 20-26 août 1953, vol. II: Épistémologie, Amsterdam, Louvain, $113-120$.

Bostrom, Nick (2003): Transhumanist Values, https://eclass.uoa.gr/modules/document/file.php/PPP566/Bostrom\%20-\% 20Transhumanist\%20Values.pdf (letzter Zugriff 13.10.2020).

Bostrom, Nick (2006): A short history of transhumanist thought, in: Analysis and Metaphysics 5/1-2, 63-95.

Bostrom, Nick/Sandberg, Anders (2008): The wisdom of nature: an evolutionary heuristic for human enhancement, in: Savulescu, Julian/Bostrom, Nick (Hg.): Human enhancement, Oxford, $375-416$.

Braidotti, Rosi (2014): Posthumanismus. Leben jenseits des Menschen, Frankfurt a. M.

Braidotti, Rosi/Hlavajova, Maria (Hg.) (2018): Posthuman Glossary, London.

Coenen, Christopher (2009): Transhumanismus, in: Eike Bohlken/Christian Thies (Hg.): Handbuch Anthropologie. Der Mensch zwischen Natur, Kultur und Technik. Stuttgart, 268-276.

Coenen, Christopher et al. (Hg.) (2010): Die Debatte über „Human Enhancement“. Historische, philosophische und ethische Aspekte der technologischen Verbesserung des Menschen. Bielefeld.

Darwin, Charles (2005): Die Abstammung des Menschen, Frankfurt a. M.

Elliott, Carl (2003): Better than well. American medicine meets the American dream, New York.

Eßmann, Boris/Bittner, Uta/Baltes, Dominik (2011): Die biotechnische Selbstgestaltung des Menschen. Neuere Beiträge zur ethischen Debatte über das Enhancement, in: Philosophische Rundschau 58/1, 1-21.

Goethe, Johann Wolfgang von (1981): Faust. Der Tragödie zweiter Teil, in: Ders.: Werke. Hamburger Ausgabe, Bd. 3, München.

Gordijn, Bert/Chadwick, Ruth (2008) (Hg.): Medical Enhancement and Posthumanity, Stuttgart. Grunwald, Armin: Technische Zukunft des Menschen? Eschatologische Erzählungen zur Digitalisierung und ihre Kritik, im vorliegenden Band.

Harari, Yuval Noah (2015): Homo Deus. A brief history of tomorrow, London.

Hauskeller, Michael (2009): Prometheus unbound. Transhumanist arguments from (human) nature, in: Ethical Perspectives 16/1, 3-20.

Hauskeller, Michael (2012): Reinventing Cockaigne. Utopian themes in transhumanist thought, in: Hastings Center Report 42/2, 39-47.

Hülswitt, Robias/Brinzanik, Roman (2010): Werden wir ewig leben?, Frankfurt a. M. 
Kurzweil, Ray (1999): The Age of Spiritual Machines. When Computers Exceed Human Intelligence, New York.

Liggieri, Kevin (2014): Zur Domestikation des Menschen. Anthropotechnische und anthropoetische Optimierungsdiskurse, Wien/Münster.

Loh, Janina (2018): Trans- und Posthumanismus zur Einführung, Hamburg.

Loh, Janina (2020): Transhumanismus und technologischer Posthumanismus, in: Heßler, Martina/Liggieri, Kevin (Hg.): Handbuch Technikanthropologie, Baden-Baden, 277-282.

Meyer-Drawe, Käte (1996): Menschen im Spiegel ihrer Maschinen, München.

Müller, Oliver (2010): Zwischen Mensch und Maschine. Vom Glück und Unglück des Homo faber, Berlin.

Müller, Oliver (2014): Prothesengötter. Zur technischen Optimierung von Menschen, in: Liebert, Wolf-Andreas et al. (Hg.): Künstliche Menschen. Transgressionen zwischen Körper, Kultur und Technik, Würzburg, 69-80.

Müller, Oliver/Liggieri, Kevin (2019): Mensch-Maschine-Interaktion seit der Antike: Imaginationsräume, Narrationen und Selbstverständnisdiskurse, in: Dies. (Hg.): Mensch-Maschine-Interaktion. Geschichte - Kultur - Ethik, Stuttgart, 3-14.

Müller, Tobias: Die transhumanistische Utopie des Mind-Uploading und die Grenzen der technischen Manipulierbarkeit menschlicher Subjektivität, im vorliegenden Band.

Nietzsche, Friedrich (2014): Also sprach Zarathustra. Kritische Studienausgabe, Bd. 4, München.

O‘Gieblyn, Meghan (2017): God in the machine: my strange journey into transhumanism, in: The Guardian, 18. April 2017, https://www.theguardian.com/technology/2017/apr/18/ god-in-the-machine-my-strange-journey-into-transhumanism (letzter Zugriff 13.10. 2020).

Pico della Mirandola, Giovanni (1988): Über die Würde des Menschen, Zürich.

Sartre, Jean-Paul (2005): Entwürfe für eine Moralphilosophie, Reinbek.

Sartre, Jean-Paul (1975): Ist der Existentialismus ein Humanismus?, in: Ders.: Drei Essays, Frankfurt a. M., 7-51.

Sloterdijk, Peter (2009): Du mußt dein Leben ändern. Über Anthropotechnik, Frankfurt a.M.

Sorgner, Stefan Lorenz (2009): Nietzsche, the Overhuman, and Transhumanism, in: Journal of Evolution and Technology 20/1, 29-42.

Sorgner, Stefan Lorenz (2019): Übermensch. Plädoyer für einen Nietzscheanischen Transhumanismus, Basel.

Tuncel, Yunus (2017) (Hg.): Nietzsche and Transhumanism: Precursor or Enemy?, Cambridge.

Vance, Ashlee (2010): Merely Human? That's So Yesterday, in: New York Times, https://www.nytimes.com/2010/06/13/business/13sing.html (letzter Zugriff 13.10.2020). 